ECCOMAS

Proceedia
COMPDYN 2019

$7^{\text {th }}$ ECCOMAS Thematic Conference on Computational Methods in Structural Dynamics and Earthquake Engineering M. Papadrakakis, M. Fragiadakis (eds.) Crete, Greece, 24-26 June 2019

\title{
OPTIMAL DISSIPATIVE COUPLING DESIGN OF TWO OSCILLATORS BASED ON NONLINEAR STOCHASTIC RESPONSE
}

\author{
Francesco Potenza ${ }^{1}$, Vincenzo Gattulli ${ }^{2}$ and Billie F. Spencer ${ }^{3}$ \\ ${ }^{1}$ Department of Civil Architectural and Environmental Engineering, University of L'Aquila \\ via G. Gronchi 18, Nucleo industriale di Pile, 67100, L'Aquila, Italy \\ e-mail: francesco.potenza@univaq.it \\ ${ }^{2}$ Department of Structural and Geotechnical Engineering, University of Rome, Sapienza \\ via Eudossiana 19, 00167, Rome, Italy \\ vincenzo.gattulli@uniroma1.it \\ ${ }^{3}$ Department of Civil and Environmental Engineering, University of Illinois at Urbana-Champaign \\ 205 North Mathews Ave, Urbana, Illinois 61801, USA \\ bfs@illinois.edu
}

\begin{abstract}
The work proposes an optimization procedure for the design of a nonlinear dissipative coupling device of two oscillators subjected to stochastic dynamic excitation. Two simple oscillators connected by Bouc-Wen type hysteretic device describe the system. Zero-mean filtered white noise is the base excitation. The knowledge of the stationary response variances, evaluated by equivalent linearization permits to define the optimization problem to design the device. Finally, Monte Carlo simulations using filtered white noise and natural earthquake records at different levels of seismic magnitude conclude the evaluation of the procedure efficacy.
\end{abstract}

Keywords: Structural optimization, nonlinear response, dissipative coupling, stochastic load 


\section{INTRODUCTION}

One of the mainly target pursued by structural design methods is to obtain a solution that assure an optimal performance. Structural optimization procedures are often proven to be an efficient tool to balance different competing design objectives [1]. Moreover, several loads that have to be taking into account in the structural design process are stochastic and dynamic in nature (like wind and seismic actions) but usually analyses related to optimization techniques are based on dynamic excitation represented by equivalent static load [2]. Many of research works on structural optimization have been driven using Monte Carlo simulations that, of course, can be time consuming and computationally demanding because several simulations are required to get converged response statistics [3]. However, scientific papers have applied random vibration theory to solve structural optimization problems. Some examples are reported in [4], [5] where the aim of the authors is to optimize elements' size for a linear five-story shear structure subject to stochastic seismic ground motion. This work propones the optimization of the nonlinear stochastic responses of a simple model composed by two linear oscillators linked by a dissipative element [6]. A depth analysis of synthetic and analytical systems could be very useful for both to explain experimental phenomena and to develop design procedures [7], [8].

\section{NONLINEAR ANALYTICAL MODEL}

Let to consider a simple nonlinear analytical model composed by two simple oscillators coupled by a nonlinear dissipative devise as reported in Figure 1. They have mass $M_{\mathrm{i}}$ and stiffness $K_{\mathrm{i}}(i=1,2)$ and, moreover, are linked together by a dissipative element. Indicating with $U_{1}$ and $U_{2}$ the relative displacements and $F$ the force delivered by the damper, the equations of motion of the two-degree-of-freedom model, shown in Figure 1, are governed by the following system:

$$
\begin{aligned}
& M_{1} \ddot{U}_{1}+K_{1} U_{1}-F=-M_{1} U_{g} \\
& M_{2} \ddot{U}_{2}+K_{2} U_{2}-F=-M_{2} U_{g}
\end{aligned}
$$

where dot indicates the derivative with respect to time $t$. Then, defining the subsequent dimensionless variables and parameters

$$
\begin{aligned}
& u_{i}=\frac{U_{i}}{L}, \quad u_{g}=\frac{U_{g}}{L}, \quad \omega_{i}^{2}=\frac{K_{i}}{M_{i}}, \quad \beta=\frac{\omega_{2}}{\omega_{1}} \\
& \rho=\frac{M_{2}}{M_{1}}, \quad f_{d}=\frac{F}{\omega_{1}^{2} M_{1} L}, \quad \tau=\omega_{1} t
\end{aligned}
$$

where $L$ is a convenient reference length, $\rho$ and $\beta$ are the mass and frequency ratio between the uncoupled oscillators, the expression of the dimensionless equations of motion written in the synthetic matrix form can be derived:

$$
\mathbf{M u}+\mathbf{K u}+\mathbf{r} f_{d}=-\mathbf{M \Gamma} \ddot{u}_{g}
$$

In such system $\mathbf{u}$ is the displacements vector while $\mathbf{M}$ and $\mathbf{K}$ are the mass and stiffness matrices. Moreover, the vectors $\mathbf{r}$ and $\Gamma$ have been introduced to allocate the control and external forces. The scalar variable $\ddot{u}_{g}$ represents the seismic ground acceleration. The form of these parameters will be the following: 


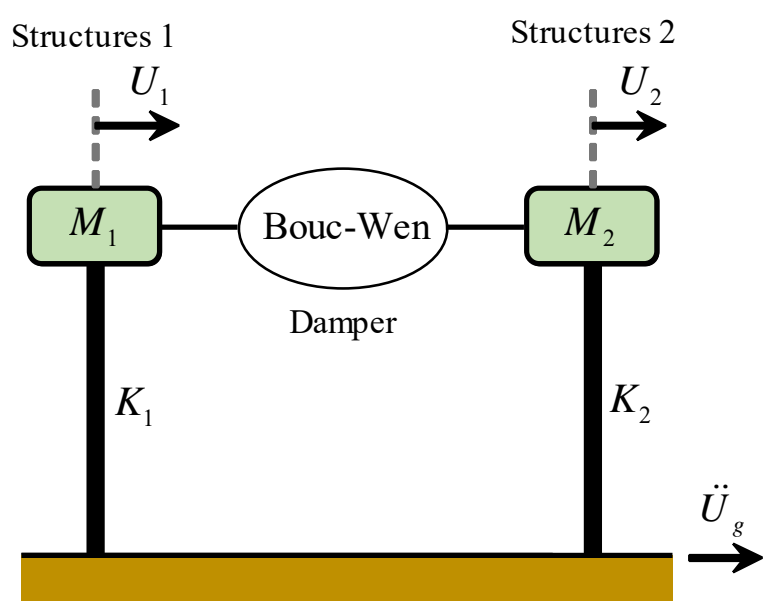

Figure 1: Nonlinear analytical model of two coupled oscillators.

$$
\mathbf{u}=\left[\begin{array}{l}
u_{1} \\
u_{2}
\end{array}\right], \quad \mathbf{M}=\left[\begin{array}{ll}
1 & 0 \\
0 & \rho
\end{array}\right], \quad \mathbf{K}=\left[\begin{array}{cc}
1 & 0 \\
0 & \rho \beta^{2}
\end{array}\right], \quad \boldsymbol{\Gamma}=\left[\begin{array}{l}
1 \\
1
\end{array}\right]
$$

After formulating the linear system, the nonlinear equation of motion can be generated considering a hysteretic behavior for the dissipative force of the damper. The new nonlinear system looks as following:

$$
\begin{aligned}
& \mathbf{M}_{\mathrm{s}} \ddot{\mathbf{u}}+\mathbf{K}_{\mathrm{s}} \mathbf{u}+\mathbf{C}_{d} \dot{\mathbf{u}}+\alpha \mathbf{K}_{d} \mathbf{u}+(1-\alpha) \mathbf{K}_{d} z=-\mathbf{M} \Gamma \ddot{u}_{g} \\
& \dot{z}=-\gamma_{b}\left|\dot{u}_{2}-\dot{u}_{1}\right| z|z|^{n-1}-\beta_{b}\left(\dot{u}_{2}-\dot{u}_{1}\right)|z|^{n}+A\left(\dot{u}_{2}-\dot{u}_{1}\right)
\end{aligned}
$$

where looking to the restoring force can be recognized two component related to the linear part modelled as a Kelvin-Voigt model (linear spring and viscous element arranged in parallel) while the nonlinear one is regulated by an adjunct variable $z$ and moreover the coefficient $\alpha$ is the post- to pre- yield stiffness ratio for the damper. The linear matrices describing the dissipative constitutive law assume the following form:

$$
\mathbf{K}_{d}=\left[\begin{array}{cc}
\eta & -\eta \\
-\eta & \eta
\end{array}\right], \quad \mathbf{C}_{d}=\left[\begin{array}{cc}
\gamma & -\gamma \\
-\gamma & \gamma
\end{array}\right]
$$

where $\eta$ and $\gamma$ are the dimensionless parameters for spring and dashpot whose form is:

$$
\eta=\frac{K}{\omega_{1}^{2} M_{1}}, \quad \gamma=\frac{C}{2 \omega_{1} M_{1}}
$$

in which $K$ and $C$ are the elastic and viscous coefficient, respectively.

The dynamic evolution of the variable $z$ is described by a Bouc-Wen model where the coefficients $\gamma_{b}$ and $\beta_{b}$ control the shape of the hysteresis loop, $A$ the restoring amplitude and $n$ the smooth transition from elastic to plastic response (for large values of $n$ the model tends to an elasto-plastic behavior).

\section{NONLINEAR STOCHASTIC RESPONSE BY EQUIVALENT LINEARIZATION}

It is well-known that for a state-space model the linear stationary stochastic response can be evaluated through the covariance matrix $\Gamma$. Indeed, assuming a system written in statespace formulation as in the following: 


$$
\dot{\mathbf{x}}=\mathbf{A} \mathbf{x}+\mathbf{B} w
$$

where $\mathrm{w}$ is a zero-mean stationary Gaussian process, A and B are the state-space matrices, $\mathrm{x}$ the state-space vector, usually composed by the displacement and velocity of the system, the stationary stochastic responses can be obtained solving the following equation [9]:

$$
\mathbf{0}=\mathbf{A} \boldsymbol{\Gamma}+\boldsymbol{\Gamma} \mathbf{A}+2 \pi \mathbf{B} S \mathbf{B}
$$

which is the well-known Lyapunov equation in the unknown covariance matrix. The variable $S$ is the power of the white noise spectral density. It right to remember that the covariance is defined as:

$$
\boldsymbol{\Gamma}_{\mathbf{x}}(t)=\mathbb{E}\left[\mathbf{x}(t) \mathbf{x}^{\mathrm{T}}(t)\right]=\left[\begin{array}{cccc}
\sigma_{x_{1}}^{2} & \mathbb{E}\left[x_{1} x_{2}\right] & \cdots & \mathbb{E}\left[x_{1} x_{n}\right] \\
& \sigma_{x_{2}}^{2} & \cdots & \mathbb{E}\left[x_{2} x_{n}\right] \\
& & \ddots & \\
\text { symm } & & & \sigma_{x_{n}}^{2}
\end{array}\right]
$$

It contains in the main diagonal the expected values of the displacements and velocities (i.e. variances and standard deviations) while out of diagonal are located the mixed expected values.

The nonlinear stochastic response can be well approximate by an equivalent linear system that will allows to easily and quickly evaluate the nonlinear solution solving the previous Lyapunov equation. The evolution of the variable $z$ can be reproduced by the following first-order differential equation:

$$
\dot{z}+C_{e q} \Delta \dot{u}+K_{e q} z=0
$$

where $\Delta \dot{u}=\dot{u}_{2}-\dot{u}_{1}$.

In [9] the authors evaluated the equivalent of $C_{e q}$ and $K_{e q}$ for Bouc-Wen model in the special case $n=1$

$$
\begin{gathered}
C_{e q}=\gamma_{b} \mathrm{E}\left[z \frac{\partial|\Delta \dot{u}|}{\partial \Delta \dot{u}}\right]+\beta_{b} \mathrm{E}[|z|]-A \\
K_{e q}=\gamma_{b} \mathrm{E}[|\Delta \dot{u}|]+\beta_{b} \mathrm{E}\left[\Delta \dot{u} \frac{\partial|z|}{\partial z}\right]
\end{gathered}
$$

Assuming a Gaussian excitation, the equivalent terms can be assessed through second moments:

$$
\begin{gathered}
C_{e q}=\sqrt{\frac{2}{\pi}}\left[\gamma_{b} \frac{\mathrm{E}(\Delta \dot{u} z)}{\sigma_{\Delta \dot{u}}}+\beta_{b} \sigma_{z}\right]-A \\
K_{e q}=\sqrt{\frac{2}{\pi}}\left[\gamma_{b} \sigma_{\Delta \dot{u}}+\beta_{b} \frac{\mathrm{E}(\Delta \dot{u} z)}{\sigma_{z}}\right]
\end{gathered}
$$

where $\mathrm{E}(\cdot)$ is the expectation operator while $\sigma$ represents the standard deviation. Moreover, expression for a general case of $n \neq 1$ can be found in Giaralis and Spanos [10]. 


\subsection{Structural responses}

Defining the state-space vector as

$$
\mathbf{x}_{\mathrm{s}}=\left[\begin{array}{lll}
\mathbf{u}^{\mathrm{T}} & \dot{\mathbf{u}}^{\mathrm{T}} & z
\end{array}\right]^{\mathrm{T}}
$$

the equivalent linear system given putting together the Eq. (5a) and Eq. (11) and subjected to a stochastic excitation assumes the following form:

$$
\begin{aligned}
& \dot{\mathbf{x}}_{\mathrm{s}}=\mathbf{A}_{\mathrm{s}} \mathbf{x}+\mathbf{B}_{\mathrm{s}} w \\
& \mathbf{y}_{\mathrm{s}}=\mathbf{C}_{\mathrm{s}} \mathbf{x}+\mathbf{D}_{\mathrm{s}} w
\end{aligned}
$$

The state-space matrices $\mathbf{A}_{\mathrm{s}}$ and $\mathbf{B}_{\mathrm{s}}$ look as:

$$
\mathbf{A}_{\mathrm{s}}=\left[\begin{array}{ccc}
\mathbf{0} & \mathbf{I} & \mathbf{0} \\
-\mathbf{M}^{-1}\left(\mathbf{K}+\alpha \mathbf{K}_{d}\right) & \alpha \mathbf{M}^{-1} \mathbf{C}_{d} & -(1-\alpha) \mathbf{K}_{d} \\
0 & -C_{e q} \mathbf{r}^{\mathrm{T}} & -K_{e q} \mathbf{r}^{\mathrm{T}}
\end{array}\right], \quad \mathbf{B}_{\mathrm{s}}=\left[\begin{array}{c}
\mathbf{0} \\
\boldsymbol{\Gamma} \\
0
\end{array}\right]
$$

The matrices related to the output $\mathbf{y}, \mathbf{C}_{\mathrm{s}}$ and $\mathbf{D}_{\mathrm{s}}$, can be defined to contain arbitrary information about the system (generally $\mathbf{D}_{\mathrm{s}}=0$ ).

Based on this linearized process, a reasonable evaluation of the nonlinear stochastic response can be carried out solving the Lyapunov equation through an iterative procedure since the equivalent coefficients, $C_{e q}$ and $K_{e q}$, depend by the standard deviations.

The robustness of this procedure has been preliminary carried out comparing the results obtained with the linearized procedure and direct integration for a nonlinear oscillator. In this case the hysteretic component of the restoring force is described through a Bouc-Wen model. The equation of motion looks as following:

$$
\begin{aligned}
& \ddot{x}+2 \zeta \omega \dot{x}+\alpha \omega^{2} x+(1-\alpha) \omega^{2} z=w(t) \\
& \dot{z}=-\gamma_{b}|\dot{x}| z|z|^{n-1}-\beta_{b} \dot{x}|z|^{n}+A \dot{x}
\end{aligned}
$$

In the below Figure 2 are illustrated two direct integration analyses related to two cases with low and high noise power. Indeed, in the Tables 1 and 2 are reported the results of the standard deviations evaluated with both methods, direct integration and linearized procedure, for
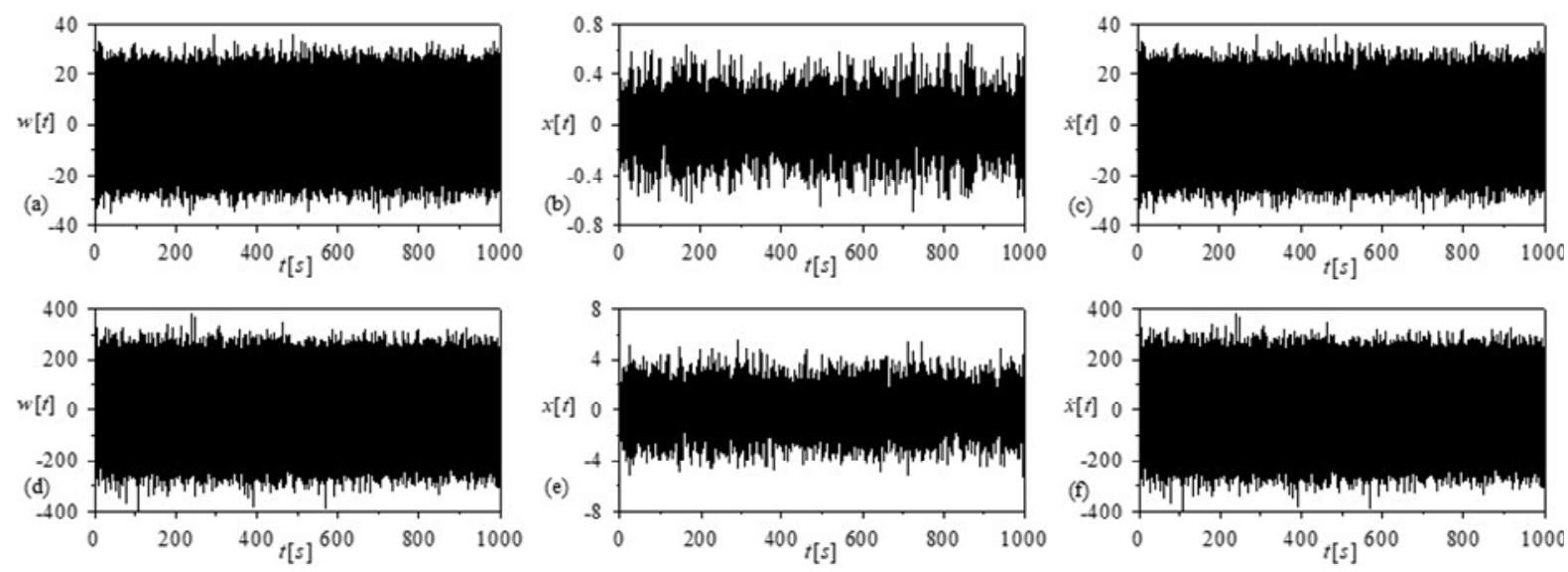

Figure 2: Direct Integration of the nonlinear oscillator. Parameters: $\omega=2 \pi 1.35, \xi=0.05, \alpha=0.05$, Bouc-Wen: $\gamma_{b}=0.5, \beta_{b}=0.5, n=1, A=1$. Noise Power: (a), (b), (c) $\mathrm{S}=0.01$; (d), (e), (f) $\mathrm{S}=1$. 


\begin{tabular}{cccccc}
\hline Method & $\mathrm{S}=0.01$ & $\mathrm{~S}=0.03$ & $\mathrm{~S}=0.10$ & $\mathrm{~S}=0.50$ & $\mathrm{~S}=1.00$ \\
\hline Direct Integration & 0.0220 & 0.0378 & 0.0671 & 0.1477 & 0.2054 \\
Linearized procedure & 0.0221 & 0.0376 & 0.0670 & 0.1440 & 0.2011
\end{tabular}

Table 1: Standard deviations of the nonlinear oscillator in terms of displacements varying the noise power S.

\begin{tabular}{cccccc}
\hline Method & $\mathrm{S}=0.01$ & $\mathrm{~S}=0.03$ & $\mathrm{~S}=0.10$ & $\mathrm{~S}=0.50$ & $\mathrm{~S}=1.00$ \\
\hline Direct Integration & 0.0220 & 0.0378 & 0.0671 & 0.1477 & 0.2054 \\
Linearized procedure & 0.0221 & 0.0376 & 0.0670 & 0.1440 & 0.2011
\end{tabular}

Table 2: Standard deviations of the nonlinear oscillator in terms of velocities varying the noise power S.

displacements and velocities. The accord appears quite good even for high values of the noise power.

\subsection{Stochastic excitation modelled as filtered white noise}

The stochastic excitation can be represented as a filtered white noise described, for example, through a Kanai-Tajimi filter:

$$
\begin{aligned}
& \ddot{x}_{f}+2 \zeta_{g} \omega_{g} \dot{x}_{f}+\omega_{g}^{2} x_{f}=w(t) \\
& \ddot{u}_{g}=\omega_{g}^{2} x_{f}+2 \zeta_{g} \omega_{g} \dot{x}_{f}
\end{aligned}
$$

The Kanai-Tajimi model is a white noise random process $w(t)$ passed through a second-order filter, $\omega_{g}$ and $\xi_{g}$ are the site natural frequency and damping of the soil. The latter are properties that depend by local site conditions, seismic hazard maps, etc. [11].

The Eq. (20) can be written in the state-space formulation:

$$
\begin{aligned}
& \dot{\mathbf{x}}_{f}=\mathbf{A}_{f} \mathbf{x}_{f}+\mathbf{B}_{f} w(t) \\
& \ddot{u}_{g}=\mathbf{C}_{f} \mathbf{x}_{f}
\end{aligned}
$$

where $\mathbf{x}_{f}$ is the filtered state vector while the matrices $\mathbf{A}_{f}, \mathbf{B}_{f}$, and $\mathbf{C}_{f}$ are chosen to represent the characteristic of the excitation.

The combined representation between system and excitation can be obtained through an augmented state vector given by

$$
\mathbf{x}_{a}=\left[\begin{array}{ll}
\mathbf{x}_{\mathrm{s}}^{\mathrm{T}} & \mathbf{x}_{f}^{\mathrm{T}}
\end{array}\right]^{\mathrm{T}}
$$

and so, combining together the equation of the structural system (Eq. 17) and the ones for the loading model (Eq. 20), both written in the state-space formulation, it is possible to get a new form of the augmented system:

$$
\begin{aligned}
& \dot{\mathbf{x}}_{\mathrm{a}}=\mathbf{A}_{\mathrm{a}} \mathbf{x}_{\mathrm{a}}+\mathbf{B}_{\mathrm{a}} w(t) \\
& \mathbf{y}_{\mathrm{s}}=\mathbf{C}_{\mathrm{a}} \mathbf{x}_{\mathrm{a}}
\end{aligned}
$$

where the new expression of the augmented state-space matrices is the following

$$
\mathbf{A}_{\mathrm{a}}=\left[\begin{array}{cc}
\mathbf{A}_{\mathrm{s}} & \mathbf{B}_{\mathrm{s}} \mathbf{C}_{\mathrm{f}} \\
\mathbf{0} & \mathbf{A}_{\mathrm{f}}
\end{array}\right], \quad \mathbf{B}_{\mathrm{a}}=\left[\begin{array}{c}
\mathbf{0} \\
\mathbf{B}_{\mathrm{f}}
\end{array}\right], \quad \mathbf{C}_{\mathrm{a}}=\left[\begin{array}{ll}
\mathbf{C}_{\mathrm{s}} & \mathbf{D}_{\mathrm{s}} \mathbf{C}_{\mathrm{f}}
\end{array}\right]
$$


The enlarged dynamical system permits to evaluate the stochastic structural response, taking into account the soil interaction.

\subsection{Structural optimization formulation}

The optimization problem is formulated as follows:

find the design variables $\boldsymbol{\theta}=\left\{\begin{array}{llll}\theta_{1} & \theta_{2} & \ldots & \theta_{N}\end{array}\right\}^{\mathrm{T}} \in \mathbf{R}^{n}$ such that it is minimized the objective function $J(\boldsymbol{\theta})$. The optimization procedure will have to respect also the requirements of a constraint function given by

$$
\left\{\begin{array}{c}
\theta_{j}>\theta_{j, \min , \quad} \quad j=1,2, \ldots N \\
\mathrm{E}\left[G_{k}\left(\mathbf{y}_{\mathrm{s}}, \boldsymbol{\theta}\right)\right] \leq 0, \quad k=1,2, \ldots m
\end{array}\right.
$$

where $\theta_{j \text {,min }}$ represents the lower bound of the jth design variable while $G_{k}\left(\mathbf{y}_{\mathrm{s}}, \boldsymbol{\theta}\right)$ is the kth constraint function in the optimization procedure.

In general, in dealing with an optimization analyses different performance competing objectives should be minimized. For example, in the control design the optimal simultaneous reduction of both displacements and absolute accelerations is a target practically impossible to reach. For this reason, in the research will be introduced a Pareto optimal front that will constitute a tool for multi-objective optimization and decision making. This optimal frontier is defined by the following expression

$$
J_{\mathrm{P}}=(1-\xi) \cdot J_{\text {dipl }}(\boldsymbol{\theta})+\xi \cdot J_{a c c}(\boldsymbol{\theta})
$$

in which $\xi$ is a parameter that provides a tradeoff between two competing objective. It will be included between 0 and 1 . When $\xi=0$ only the optimization of the displacements will be take into account while for $\xi=1$ will be optimize only the accelerations. When $\xi \in(0,1)$ the resulting target will be a linear combination of both performance objectives.

\section{CONCLUSIONS}

The paper propones an iterative procedure to evaluate the nonlinear response of a simplified model subject to stochastic excitation. The procedure is also suitable for a filtered white noise excitation that takes into account the soil interaction. The main findings permit to develop a design method for hysteretic device based on multi-objective optimization based on a Pareto optimal frontier.

\section{ACKNOWLEDGEMENT}

The presented research results have received funding by the Italian Department of Civil Protection under the project DPC-ReLUIS 2014-2016 and Italian Government under Cipe resolution n.135 (Dec. 21, 2012), project INnovating City Planning through Information and Communication Technologies.

\section{REFERENCES}

[1] J. Xu, B.F. Spencer Jr., X. Lu, Performance-based optimization of nonlinear structures subject to stochastic dynamic loading. Engineering Structures, 134, 334-345, 2017. 
[2] M. Al-Ansari, A. Senouci, Drift optimazation of high-rise buildings in earthquake zones. The Structural Design of Tall and Special Buildings, 20, 208-222, 2011.

[3] R.J. Balling, L.J. Balling, P.W. Richards, Design of buckling-restrained braced frames using nonlinear time history analysis and optimization, Journal of Structural Engineering, 135(5), 461-468, 2009.

[4] H.A. Jensen, A.E. Sepulveda, Optimal design of uncertain system under stochastic excitation, AIAA Journal, 38(11), 2133-2141, 2000.

[5] H.A. Jensen, Structural optimization of non-linear systems under stochastic excitation, Probabilistic Engineering Mechanics, 21(4), 397-409, 2006.

[6] V. Gattulli, F. Potenza, B.F. Spencer, Design criteria for dissipative devices in coupled oscillators under seismic excitation. Structural Control and Health Monitoring, e2167, 2018 .

[7] A.M. Ceci, V. Gattulli, F. Potenza, Serviceability and damage scenario in irregular RC structures: post-earthquake observations and modelling predictions. Journal of Performance of Constructed Facilities, 27(1), 95-115, 2013.

[8] V. Gattulli, M. Lepidi, F. Potenza, U. Di Sabatino, Dynamics of masonry walls connected by a vibrating cable in a historic structure, Meccanica, 51(1), 2813-2826, 2016.

[9] Y.K. Wen, Equivalent Linearization for Hysteretic System Under Random Excitation, Journal of Applied Mechanics, 47, 150-154, 1980.

[10] A. Giaralis, P.D. Spanos, Derivation of equivalent linear properties of Bouc-Wen hysteretic systems for seismic response spectrum analysis via statistical linearization. $10^{\text {th }}$ HSTAM International Congress on Mechanics, Chania, Greece, May 25-27, 2013.

[11] S.P. Lai, Statistical characterization of strong ground motion using power spectral density function. Bulletin of Seismological Society of America, 72(1):259-274, 1982.

[12] K. Miettinen, Nonlinear multiobjective optimization. Springer Science \& Business Media, 2012. 